\title{
Magnetospheric Radio Emissions from Exoplanets with the SKA
}

\author{
Philippe Zarka*1, T. Joseph W. Lazio",3, Gregg Hallinan ${ }^{3}$, \& "Cradle of Life” WG \\ ${ }^{1}$ LESIA, CNRS - Obs. Paris, Meudon, France; ${ }^{2} J P L ;{ }^{3}$ Caltech, Pasadena, CA, USA \\ E-mail: philippe.zarka@obspm.fr
}

Planetary-scale magnetic fields are a window to a planet's interior and provide shielding of the planet's atmosphere and surface for life. The Earth, Mercury, Ganymede, and the giant planets of the solar system all contain internal dynamo currents that generate planetary-scale magnetic fields. When coupled to energetic ( $\mathrm{keV})$ electrons, such as those produced by solar windmagnetosphere interaction (compression or magnetic reconnection), magnetosphere-ionosphere or magnetosphere-satellite coupling, the polar regions of a planetary magnetic field are the place of intense, coherent, circularly polarized cyclotron radio emissions. These emissions - that may be as intense as solar ones - are produced by all magnetized planets in the solar system in the $\mathrm{MHz}$ range, and up to $40 \mathrm{MHz}$ at Jupiter. Detection of similar emissions from exoplanets will provide constraints on the thermal state, composition, and dynamics of their interior - very difficult to determine by other means - as well as an improved understanding of the planetary dynamo process and of the physics of star-planet plasma interactions.

Detailed knowledge of magnetospheric emissions from solar system planets and the discovery of exoplanets motivated both theoretical and observational work on magnetospheric emissions from exoplanets. Scaling laws and theoretical frameworks were built and extrapolated to obtain orderof-magnitude predictions of frequencies and flux densities of exoplanetary radio emissions. The present stage of the theory suggests that radio detection of exoplanets will develop the new field of comparative exo-magnetospheric physics, but also permit to measure exoplanetary parameters such as rotation or orbit inclination. Observational searches started even before the confirmed discovery of the first exoplanet.

We review the scientific return of the detection of exoplanetary radio emissions, the current status of observational searches, and discuss the future promise in the context of SKA, especially SKA1LOW. To the extent that Jupiter's magnetic field is not exceptionally strong, the current lower frequency limit of $50 \mathrm{MHz}$ implies that SKA1-LOW will likely detect Jovian-mass planets. With the currently planned sensitivity of SKA1-LOW, we estimate that a Jupiter-like planet could be detected to about $10 \mathrm{pc}$. Within this volume there are $\sim 200$ known stars and $\sim 35$ currently known exoplanets, and this number should increase substantially with coming space missions dedicated to transits and powerful ground-based instruments. The accessible volume will be much increased if scaling laws derived in our solar system can be reliably extrapolated to exoplanetary systems, permitting to measure lower mass planets' dynamos and magnetospheres.

Advancing Astrophysics with the Square Kilometre Array

June 8-13, 2014

Giardini Naxos, Sicily, Italy

* Speaker. 


\section{Introduction}

Planets appear to be the most favourable cradle of life in the context of present astrobiology. With the growing number of exoplanets ( $>1800$, cf. www.exoplanet.eu) detected by radial velocity or transits, which cover a broad range of sizes, masses and orbital parameters, it is increasingly desirable to determine the physical properties of exoplanetary systems to carry in-depth comparative studies. Topics of interest include magnetospheric dynamics, energetics of star-planet interactions, planetary rotation, planetary dynamos (large-scale planetary magnetic fields play an important role in protecting the planet's surface and atmosphere), etc.

Experience from our solar system planets and theory tell us that the most intense electromagnetic emissions from magnetospheres result from coherent cyclotron radiation, through which $\mathrm{keV}$ electrons in strong magnetic fields generate intense, circularly polarized, sporadic emissions in the $\mathrm{MHz}$ to tens of $\mathrm{MHz}$ range (synchrotron emission is also produced at higher frequencies, but with a much lower intensity). Measuring the dynamic spectra in intensity and (circular) polarization of these cyclotron emissions can give access to the above physical parameters, as well as orbit inclination and the presence of satellites.

In section 2, we summarize our observational and theoretical knowledge of solar system magnetospheres and radio emissions, review the drivers for electron acceleration (star-planet or stellar wind-planet interactions, and magnetosphere-ionosphere or magnetosphere-satellite coupling), and extrapolate them to various exoplanetary conditions. In section 3, we summarize past and ongoing observations. Non detections put a stringent limit on planetary emission above $\sim 150 \mathrm{MHz}$, and hints of detections remain to be confirmed. In section 4, we examine the prospects for SKA observations, from early science operations through Phase 1 to Phase 2. In section 5, we give a special attention to synergies, both with other SKA observations and with observations at other wavelengths.

\section{Background and Motivation}

\subsection{Solar system magnetospheres and radio emissions}

The Earth, Mercury, Ganymede, and the giant planets of the solar system all contain internal dynamo currents that generate planetary-scale magnetic fields. These internal dynamo currents arise from differential rotation, convection, compositional dynamics, or a combination of these in objects' interiors. Interaction of these planetary-scale magnetic fields with the solar wind creates a magnetosphere, a cavity in the wind dominated by the planet's magnetic field. Inside the cavity, charged particles are accelerated to $\mathrm{keV}-\mathrm{MeV}$ energies by various processes (magnetic reconnection at the boundaries, interaction with embedded moons, waves...).

Accelerated charged particles in a magnetic field generate radio emissions. The most intense ones are produced at high magnetic latitudes (so-called auroral, circumpolar regions) by a nonthermal coherent process well-identified today, that involves keV electrons: the Cyclotron Maser Instability (CMI) (Treumann 2006). This process widely operates at all magnetized planets, which are thus strong radio emitters (Zarka 1998). Emitted frequencies are local cyclotron frequencies met by energetic electrons moving along magnetic field lines. Their range extend from very low values (a few $\mathrm{kHz}$ ) far from the planet, up to the maximum surface cyclotron frequency $\left(\mathrm{f}_{c e}[\mathrm{MHz}]\right.$ 
$=2.8 \mathrm{~B}[\mathrm{G}] ; 1 \mathrm{G}=10^{-4} \mathrm{~T}$ ). For most solar system planets, planetary surface field magnitude is $\leq 1 \mathrm{G}$, thus emitted frequencies lie below $\sim 2 \mathrm{MHz}$. They are reflected off by the Earth's ionosphere and thus cannot be detected from the ground. Jupiter, having a stronger dynamo and surface field (up to $14 \mathrm{G}$ ), emits CMI radiation up to $40 \mathrm{MHz}$, which consists of bursts of duration from sub-millisecond to several tens of minutes. These bursts are easily detected by ground-based radiotelescopes.

$\mathrm{MeV}$ electrons also produce synchrotron emission in Jupiter's inner magnetosphere (so-called radiation belts) (de Pater 2004). Its spectrum extends over high harmonics of the cyclotron frequencies, up to several GHz. The synchrotron process is a nonthermal incoherent mechanism, much less efficient than the CMI, so that this emission is $10^{5}$ times weaker than the CMI. It is also much more stable in time, showing variations at timescales longer than days/weeks.

\subsection{Predictions for radio emissions from exoplanets}

Due to the high efficiency of the CMI, Jupiter's auroral radio emissions are extremely intense (brightness temperature $\geq 10^{19} \mathrm{~K}$, flux density $\geq 10^{7} \mathrm{Jy}$ at Earth (Zarka 2004)), as intense as solar ones (which are mostly due to plasma radiation), as shown on Figure 1. Considering this favourable planet-star contrast $\sim 1$, much larger than in the visible $\left(\sim 10^{-9}\right)$ or infrared $\left(\sim 10^{-6}\right)$ ranges, observational searches for low frequency radio emission from exoplanets started even before the confirmed discovery of any exoplanet (Zarka 2011, and references therein). It proved to be a difficult task and no unambiguous detection has been obtained yet (see below). But the discovery of many exoplanets by radial velocities and transit methods has motivated both theoretical and observational work on magnetospheric emissions from exoplanets.

It was soon realized that due to the high brightness of the galactic radio background, Jupiter's decametric CMI emission cannot be detected at stellar distances (several pc) even with the largest existing instruments. The search for more intense planetary emissions followed two complementary directions: (i) establishing empirical scaling laws for radio emissions from solar system planets and extrapolating them to exoplanets, and (ii) identifying the primary engine of planetary radio emissions and the regime of parameters which allow this engine to produce more powerful emissions.

A scaling law was first found to relate the average magnetospheric radio output with the total solar wind power input (dominated by the solar wind ram pressure) on the magnetospheric cross section (Farrell et al. 1999), with a proportionality constant $\sim 10^{-5}$. The specific case of coronal mass ejections (CME) impacting a magnetosphere was also considered (Grießmeier et al. 2007). A second scaling law was found to relate the same radio output with the incident Poynting flux convected by the solar wind on the magnetospheric cross section (Zarka et al. 2001, Grießmeier et al. 2007), with a proportionality constant $\sim$ a few $\times 10^{-3}$, as illustrated in Figure 2 . This scaling law was found to apply more generally to any plasma flow-obstacle interaction (contrary to the scaling law based on total power input), including Io-Jupiter and Ganymede-Jupiter interactions, and at least to one documented example of magnetized binary star (the RS CVn system V711 $\tau$ ) (Zarka 2010, and references therein).

The physical mechanisms underlying these scaling laws include magnetospheric compressions by the solar wind or CME and associated shocks, magnetic reconnection between the magnetic 


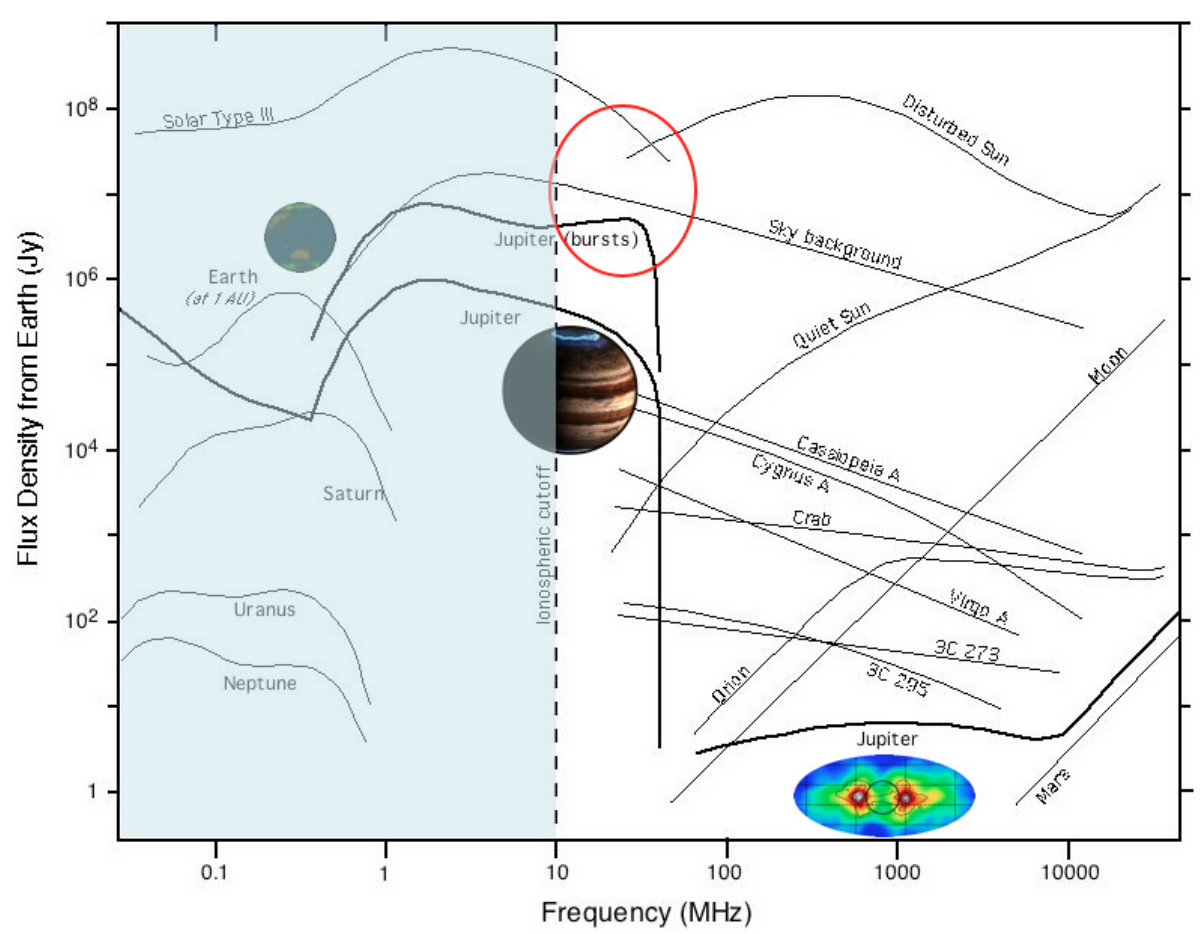

Figure 1: Spectra of astronomical radiosources detected from the Earth's vicinity. Auroral planetary spectra lie below the Earth's ionospheric cutoff, except Jupiter's decametric (CMI, auroral and Io-induced) and metric-decimetric (synchrotron) emissions. Normalized to the same observer distance of 1 AU, Jupiter's spectrum must be upscaled by $\times 20$, Saturn's by $\times 100$, Uranus' by $\times 400$, and Neptune's by $\times 900$, so that all are grouped within 2-3 orders of magnitude.

fields of the flow and of the obstacle, and Alfvén waves produced by the so-called "unipolar inductor" interaction of a conducting non-magnetized body (the moon Io) moving across magnetic field lines (of Jupiter). All these mechanisms lead to the acceleration of electrons that in turn emit radio waves. Jardine \& Cameron (2008) proposed direct electron acceleration at the magnetosphere's nose through a runaway mechanism. Nichols (2011) estimated the power dissipated by magnetosphere-ionosphere coupling, independent of the solar wind.

For exoplanets orbiting very close to their parent star, especially hot jupiters, it was found that star-planet magnetic/plasma interactions (SPI - incl. magnetospheric compression, magnetic reconnection, and unipolar inductor) involve a power $10^{3}$ to $10^{6}$ times larger than that of the solar wind-Jupiter interaction (Zarka 2007). In the unipolar inductor case, the star needs to be strongly magnetized (surface field $\geq 10^{1-2} \mathrm{G}$ ), the exoplanet plays the role of Io and its parent star that of Jupiter. Extrapolating the above scaling laws, their radio output should accordingly be $10^{3}$ to $10^{6}$ times that of Jupiter. Willes \& Wu (2005) studied the particular case of terrestrial planets in close orbits around magnetic white dwarf stars, acting as a unipolar inductor, and generating CMI radio emission with large flux densities. These systems could be remnants of main sequence stars with a planet surviving the stellar expansion phase and back in stable orbit.

For exoplanetary magnetospheres governed by internal processes (magnetosphere-ionosphere coupling), it was found that for giant planets orbiting at a few AU from their parent star, the auroral 


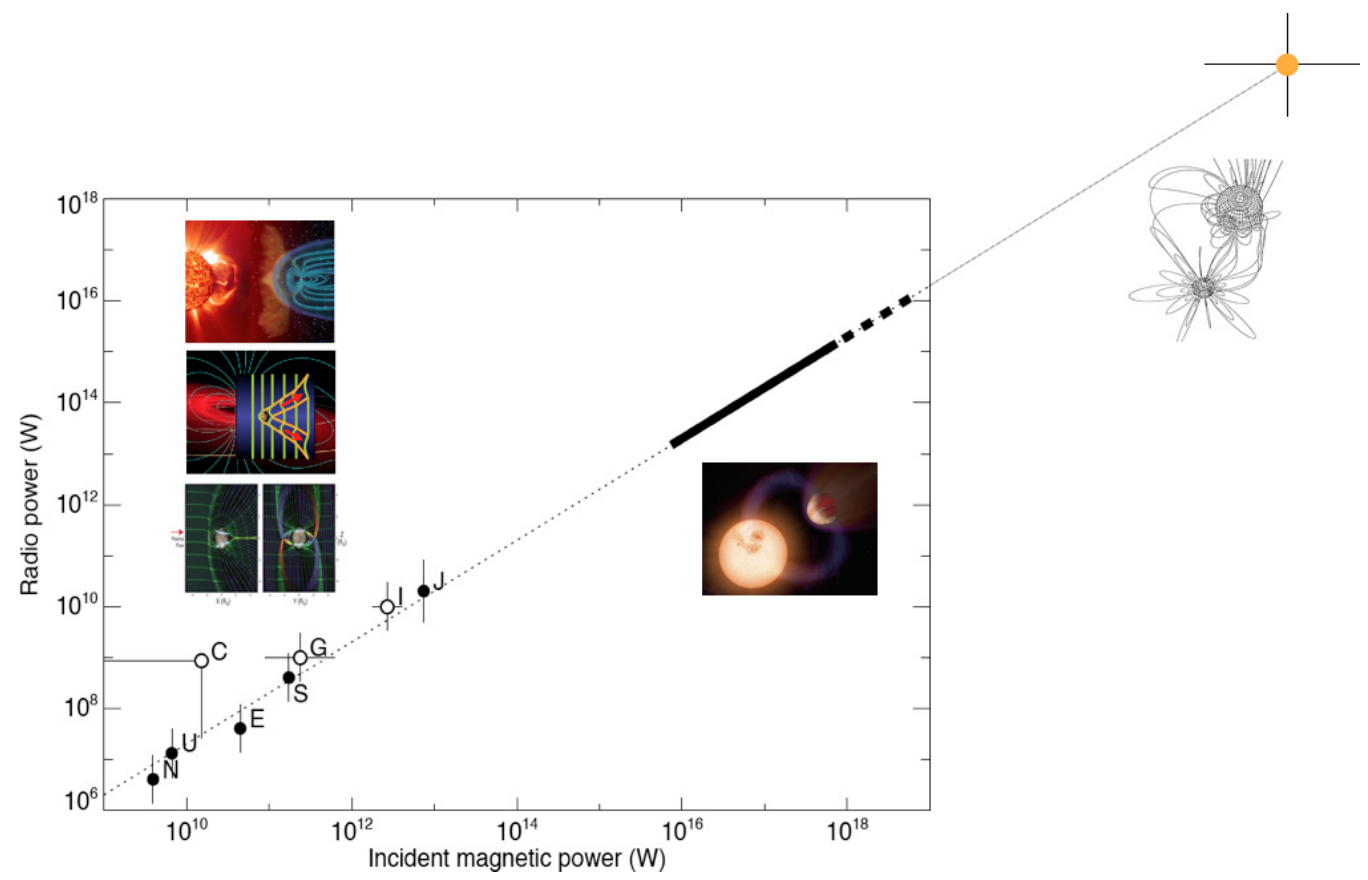

Figure 2: Scaling law relating magnetospheric (Earth, Jupiter, Saturn, Uranus and Neptune) and satelliteinduced (Io, Ganymede, Callisto) average radio power to incident Poynting flux of the plasma flow on the obstacle. Dashed line has slope 1, emphasizing the proportionality between ordinates and abscissae, with a coefficient $\sim 2 \times 10^{-3}$. Note that planetary radio bursts can reach $10 \times$ (resp. $100 \times$ ) the average value $\sim 10 \%$ (resp. $\sim 1 \%$ ) of the time. The thick bar extrapolates to hot jupiters the magnetospheric interaction (solid) and satellite-planet electrodynamic interactions (dashed). The orange dot illustrates the case of the RS CVn magnetic binary V711 $\tau$ discussed in the text. Insets sketch the types of interaction.

radio output may reach $10^{4}$ to $10^{5}$ times that of Jupiter if the star has a strong X-UV output and the planet has a fast rotation ( $\mathrm{P}=1-3$ hours).

All theoretical predictions thus suggest that exoplanetary magnetospheric radio emissions much stronger than Jupiter's are likely to exist, and thus be detectable at stellar distances with large enough instruments. Sporadic further amplifications may occur e.g. due to scintillations. CMI radio emission generated within the exoplanet's magnetosphere is likely to be restricted to low frequencies (10's of MHz, depending on the planetary field strength), whereas unipolar inductor driven CMI emission, being excited by the exoplanet in the stellar magnetic field, may reach higher frequencies (100's MHz to a few $\mathrm{GHz}$ ).

Finally, it must be noted that CMI radiation is strongly circularly/elliptically polarized (synchrotron emission from Jupiter's radiation belts is linearly polarized), and beamed anisotropically so that it is always deeply modulated by the planetary rotation. Conversely, solar/stellar plasma radiation is essentially unpolarized and sporadic. Even in the absence of sufficient angular resolution (known exoplanets orbit at $\ll 1$ " from their parent star), polarization and temporal modulations should allow to discriminate stellar and exoplanetary radio emissions, as demonstrated by the simulations presented in the next section. Theoretical predictions thus suggest that detection and identification of CMI radio emission from exoplanets is possible, so that it is worth observing. But what do we have to learn through radio emission detection? 


\subsection{Motivations for the search for exoplanet's radio emissions}

Planetary magnetic fields are a window to a planet's interior, and magnetospheric radio emissions are a probe of planetary magnetic fields. Ground-based detection of Jupiter's decametric radio emission (Burke \& Franklin 1955) provided the first proof of existence and the first measurement of the Jovian magnetic field. Its monitoring allowed one to define accurately the rotation of its interior, and to discover the existence of a strong interaction between the moon Io and the Jovian magnetosphere (Bigg 1964). Maps of the synchrotron emission revealed the tilt of the magnetic field relative to the rotation axis $\left(\sim 10^{\circ}\right)$ (Berge \& Gulkis 1976). Magnetospheric radio emissions are thus a unique tool for probing exoplanet's interior structure (composition, thermal state) and dynamics (including the effect of spin-orbit locking for hot jupiters), leading to an improved understanding of the planetary dynamo process.

Each planetary magnetosphere - in our solar system - is a space plasma physics laboratory. In-situ spacecraft exploration and remote electromagnetic (Radio, UV, IR, X) observations permitted to infer their general properties, but also revealed numerous specificities related to the magnetic field amplitude, tilt, distance to Sun, presence of embedded moons and rings, atmospheric composition...

The CMI theory provides a well-understood, quantitative framework for studying magnetospheric radio emissions properties and using them as a diagnostic tool of planetary plasma environments and processes. Recent simulations in that framework (Hess \& Zarka 2011) showed that the measurement of the radio dynamic spectrum - in intensity and polarization - of an exoplanetary system, in addition to allowing us to discriminate between stellar and exoplanetary emissions, would bring the following informations (difficult or impossible to determine by other means) about these objects, as illustrated in Figure 3: in addition to the exoplanet's magnetic field intensity and tilt, one will be able to measure the planetary rotation and revolution periods (effectively testing tidal spin-orbit synchronization), the orbit inclination (resolving the ambiguity on the planet's mass), and identify the primary engine of the electromagnetic output of the system: SPI (magnetospheric compression, stellar wind-magnetosphere magnetic reconnection, or "unipolar inductor" interaction if the exoplanet is weakly magnetized and the star strongly magnetized), or internal magnetospheric processes (magnetosphere-ionosphere or magnetosphere-moon coupling). Temporal modulations different from the planetary rotation and orbital periods could even reveal the presence of moons or the signature of the stellar wind activity.

Similar to the question of solar system formation, which requires comparison of numerous systems to infer general properties, the recent field of comparative magnetospheric physics (which relies on a corpus of 6 very different planetary magnetospheres so far) will considerably expand with the detection of exoplanetary radio emissions, that will permit to explore a large range of parameters (star-planet distance, stellar magnetic field and wind strength, stellar X-UV flux, planetary magnetic field, rotation, orbit inclination... ), better understand the diversity of planetary dynamos, study interactions between plasma flows (magnetized or not) and obstacles (magnetized or not) in various regimes, and infer the energy dissipation involved in SPI. Furthermore, the above scaling laws will be confirmed and/or modified or refined, expanding their range of application and their predicting capability.

Existence of a magnetosphere is also an essential ingredient in favour of the possibility to de- 

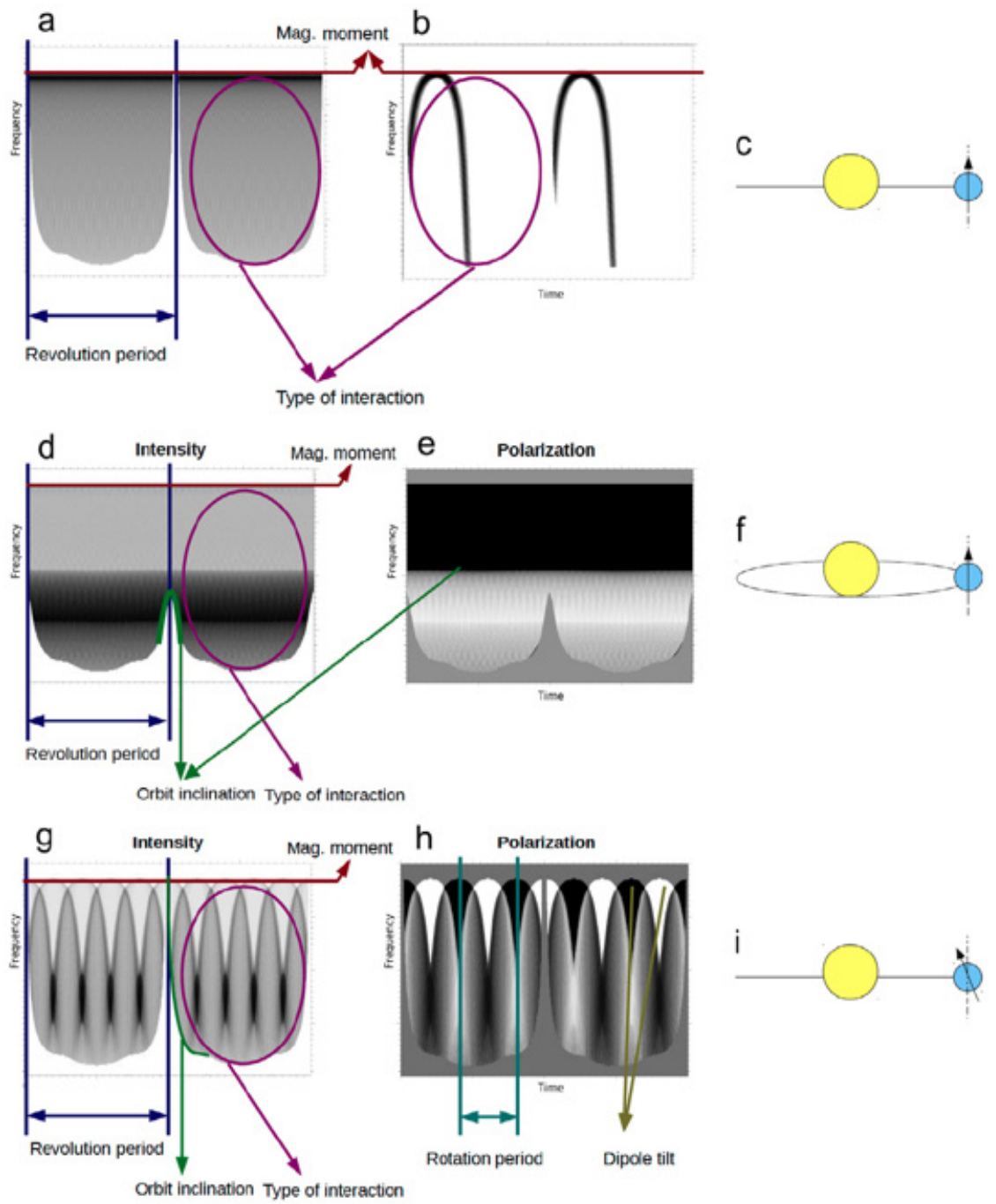

Figure 3: Simulated dynamic spectra in intensity $(a, b, d, g)$ and circular polarization $(e, h)$ and associated parameters of the system that can be determined: $(a, b, c)$ exoplanetary magnetic field aligned with the rotation axis and $0^{\circ}$ orbit inclination, for emission (a) of a full exoplanet's auroral oval and (b) an auroral active sector fixed in local time. (d,e,f) aligned exoplanetary magnetic field and $15^{\circ}$ orbit inclination, for emission of a full auroral oval. (g,h,i) exoplanetary magnetic field tilted by $15^{\circ}$ and $0^{\circ}$ orbit inclination, for emission of a full auroral oval. From (Hess \& Zarka 2011).

velop life: it ensures shielding of the planet's atmosphere and surface, preventing $\mathrm{O}_{3}$ destruction by cosmic rays bombardment as well as atmospheric erosion by the stellar wind or CMEs. It also limits atmospheric escape, as ionized material following dipolar field lines returns to the atmosphere (Grießmeier et al. 2004, 2005). Finally, radio detection may evolve as an independent discovery tool, complementary to radial velocities or transit measurements because it is more adapted to finding planets (giant or terrestrial) around active, magnetic or variable stars. 

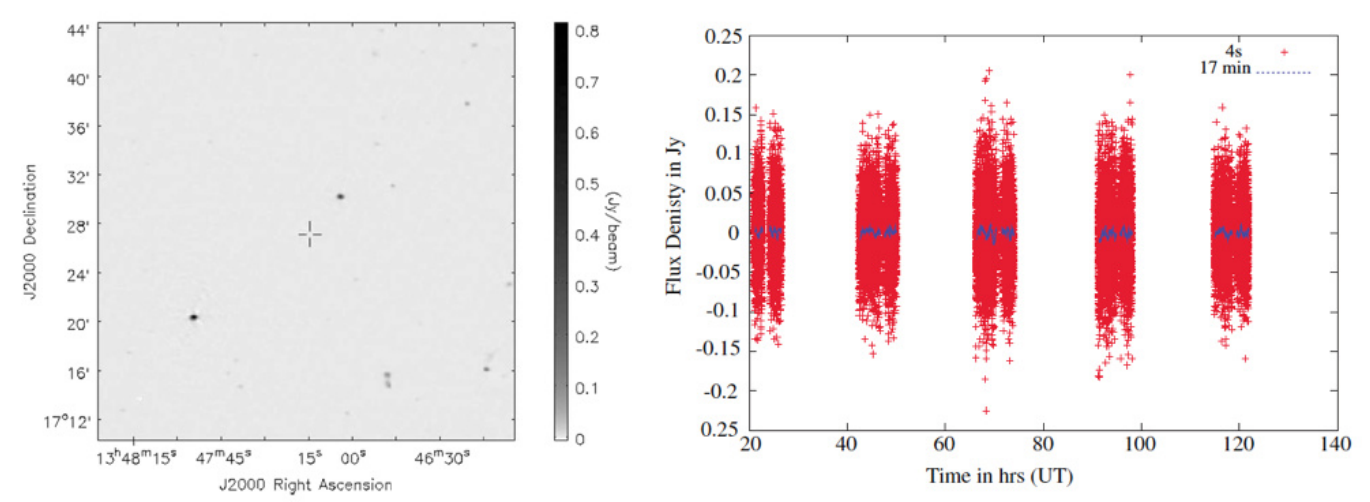

Figure 4: (left) $153 \mathrm{MHz}$ GMRT image of the $\tau$ Boo field integrated over 40 hours. The crosshairs indicate the position of the star (and planet). The rms noise in $400 \mu \mathrm{Jy}$ near the position of the undetected planet. (right) Light curves for the right circularly polarized flux at the position of $\tau$ Boo over the 40 hours of observation. Time series at time resolution of $4 \mathrm{sec}$ (red) and $17 \mathrm{~min}$ (blue) have rms noise of $65 \mathrm{mJy}$ and 4 mJy respectively. No evidence of emission is present at any observed orbital/rotational phase of the planet. From (Hallinan et al. 2013).

\section{Past and ongoing observations and results}

Most of the searches focussed on known exoplanetary systems discovered by radial velocity or transits measurements. Application of scaling laws to the exoplanet census (e.g. from exoplanet.eu) guided target selection (Lazio et al. 2004, Grießmeier et al. 2007, 2011), with promising candidates such as $\tau$ Boo, $v$ And or $55 \mathrm{Cnc}$. Other selection criteria included the existence of a strong stellar magnetic field, measured by Zeeman-Doppler imaging (as for the transiting planet HD 189733) (Farès et al. 2010), planets with a very elliptical orbit and a periastron very close to the star (HD 80606), or planets for which SPI was tentatively detected via optical measurements (HD 179949, $v$ And), although the sporadicity of observed signatures makes them not fully convincing (Shkolnik et al. 2008, and references therein).

Observations at the VLA provided no detection of $\tau$ Boo at $74 \mathrm{MHz}$ with $\sim 100 \mathrm{mJy}$ sensitivity (Farrell et al. 2003, 2004), or of HD 80606 at $330 \mathrm{MHz}$ and $1465 \mathrm{MHz}$ with resp. $1.7 \mathrm{mJy}$ and 0.048 mJy sensitivities (Lazio \& Farrell 2007, Lazio et al., 2010a). Negative results were also obtained with the GMRT at 153, 244 and $614 \mathrm{MHz}$ on HD 189733 (Lecavelier et al. 2009, 2011) with resp. $2.1 \mathrm{mJy}, 2 \mathrm{mJy}$ and $0.16 \mathrm{mJy}$ sensitivities, and at $153 \mathrm{MHz}$ on various targets ( $v$ And, $\varepsilon$ Eri, HD 128311) (Winterhalter et al. 2006, George \& Stevens 2007, 2008). A recent very significant negative result, shown in Figure 4, comes from the deepest $150 \mathrm{MHz}$ observation of $\tau$ Boo at GMRT, for $40 \mathrm{~h}$ (half of the exoplanet's orbital period) covering all orbital/rotational phases, that provided no detection at $\sim 1$ mJy level, setting this flux density level as an upper limit for both quiescent and rotationally modulated radio emission from a hot Jupiter (Hallinan et al. 2013). No detection either was obtained on a dozen targets observed during a few hours each at UTR-2 between $\sim 15$ and $30 \mathrm{MHz}$, specifically looking for pulses shorter than $\sim 1 \mathrm{~min}$., for which statistical data accumulation led to a sensitivity between $100 \mathrm{mJy}$ and $1500 \mathrm{mJy}$ depending on the target (Zarka et al. 1997, Ryabov et al. 2004).

These non detections, summarized in Figure 5, can be due to the absence of significant emis- 
sion from these targets (permanent of at observation epochs, due to their intrinsic variability), to magnetic dipole moments of the observed exoplanets too weak to generate a surface magnetic field permitting CMI emission to occur at the observed frequencies ( $\mathrm{B}_{\text {surface }}>26 \mathrm{G}$ at $74 \mathrm{MHz}, \mathrm{B}_{\text {surface }}$ $>54 \mathrm{G}$ at $150 \mathrm{MHz} .$. ), or to the Earth lying outside the beaming pattern of the planet's radio emission at the time of observation (CMI indeed produces narrowly beamed emission at specific angles relative to the magnetic field in the source; this beaming strongly affects the visibility of the emission as a function of time). In the case of UTR-2, the frequency range matches the decametric emission from Jupiter but the sensitivity was moderate.

More encouraging results were obtained at two occasions with the GMRT: (i) a $\sim 3.9$ mJy emission was detected in 2009 at $153 \mathrm{MHz}$ at $<1$ FWHM beam distance from the system HATP-11 that includes a Neptune-mass transiting exoplanet, and the light curve was consistent with an eclipse when the planet passed behind the star (Figure 6); but an equally sensitive observation repeated in 2010 did not detect any significant signal in the radio light curve near the same position, suggesting a variable planetary emission or a false positive (5\% probability) in 2009 (Lecavelier et al. 2013). (ii) The first published part of the ongoing GMRT sky survey (TGSS) at $150 \mathrm{MHz}$ encompasses 175 exoplanetary systems; for 4 of them (61 Vir, 1RXS1609, HD 86226, and HD 164509) TGSS radio sources were found coinciding with or located very close to their coordinates, with flux densities of $\sim 120,28,19$, and 18 mJy respectively (Sirothia et al. 2014). For the latter two, detection is marginal and should be confirmed by deeper observations. For all four ones, further observations are needed to exclude possible coincidence with a background radio source. The authors do not claim any unambiguous detection, but this may be the top of the iceberg showing up. For the other 171 exoplanetary systems, TGSS non detection provides $3 \sigma$ upper limits between $8.7 \mathrm{mJy}$ and $136 \mathrm{mJy}$.

Ongoing targeted searches are carried on (by the authors of this chapter) with UTR-2 and LOFAR, several tens of hours being distributed on a few dozen targets (a few hours per target, and recently 32 hours on $55 \mathrm{Cnc}$ ). More extensive observations are carried out with the LWA in the frame of the HJUDE project, aiming at thousands of observing hours distributed on a few targets (incl. $\tau$ Boo) (Hartman et al. 2013). UTR-2 has the advantage of a large effective area $(\sim 150000$ $\mathrm{m}^{2}$ ) and observes at the lowest frequencies accessible from the ground (10-32 MHz), but measures a single linear (EW) polarization and has to deal with a polluted radio spectrum. LOFAR is the largest present radio telescope (interferometer) in the range $30-250 \mathrm{MHz}$, that may extend down to $10 \mathrm{MHz}$ with reduced sensitivity (van Haarlem et al. 2013). Exoplanet search is conducted in its low band ( $\sim 20-80 \mathrm{MHz})$, in full polarization. HJUDE relies on a relatively small instrument (256 dipoles) in the 10-88 MHz band, but with a large duty cycle (searching for periodic signals at exoplanet's orbital periods) and full polarization measurements. Data analysis is ongoing in all cases.

Systematic searches in survey data will continue in the next releases of the TGSS, and is starting in LOFAR's Multifrequency Snapshot Sky Survey (Heald et al. 2014). The latter is a northern sky survey in the spectral ranges $30-75 \mathrm{MHz}(8 \times 2 \mathrm{MHz}$ bands, $\leq 100$ " resolution, $\leq 15$ $\mathrm{mJy} / \mathrm{beam})$ and $120-160 \mathrm{MHz}(8 \times 2 \mathrm{MHz}$ bands, $\leq 120$ " resolution, $\leq 5 \mathrm{mJy} / \mathrm{beam})$. The highfrequency part is near complete whereas the low-frequency part is ongoing.

We are thus in a situation where efforts intensify, both for targeted observations (still at a modest level with large instruments) and systematic searches in sky surveys (that become available 


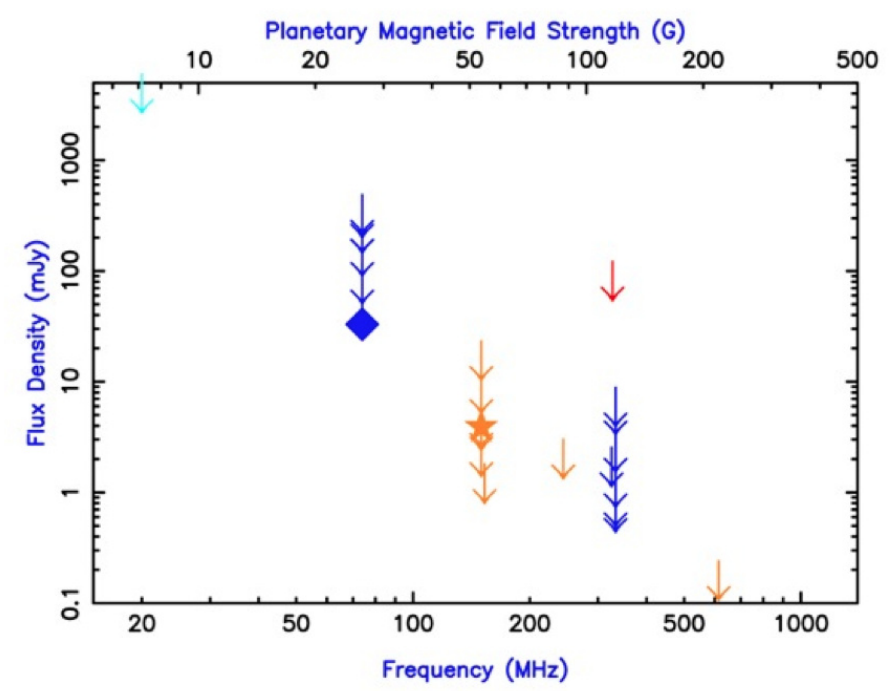

Figure 5: Summary of previous attempts to detect radio emissions from exoplanets. Blue symbols are from the VLA, red from the GBT, orange from the GMRT, and cyan from UTR-2. Arrows are upper limits from various targeted observations. The blue diamond is the statistical upper limit from Lazio et al. (2010b) on the average emission from potential planets in the solar neighbourhood. The orange star is the unconfirmed detection from Lecavelier et al. (2013).

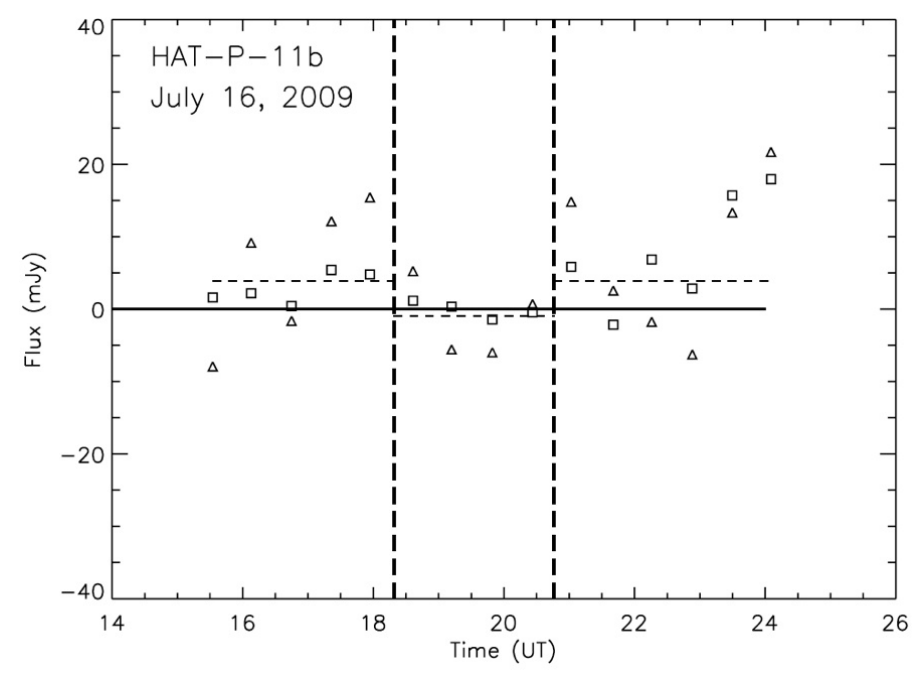

Figure 6: Time series of the $150 \mathrm{MHz}$ flux density measured on July 16, 2009, in the direction of the radio source near HAT-P-11. Measurements have been rebinned to $36 \mathrm{~min}$. Triangles and squares correspond to the right and left circular polarizations. The two vertical - dashed lines indicate the beginning and the end of the planet's eclipse behind the host star. The dashed horizontal lines show the box-shaped eclipse light curve fitted to the data points. From (Lecavelier et al. 2013). 
for the first time at low enough frequencies). First hints of detection are encouraging but not yet unambiguous.

\section{Science outcome enabled by SKA}

The result of past searches suggest that magnetospheric radio emissions much stronger than Jupiter's and at frequencies $\geq 150 \mathrm{MHz}$ are rare. It is thus needed to explore a large sample of targets with highest possible continuum sensitivity, preferably at low frequencies (although unipolar inductor driven CMI emission may reach hundreds of $\mathrm{MHz}$ ). At the SKA angular resolution ( $\sim 11$ " for SKA1-LOW at $110 \mathrm{MHz}, \sim 1$ " for SKA1-SUR and SKA1-MID at 0.5-1 GHz), it will generally not be possible to resolve exoplanet-star systems. Once a signal is detected, attributing it to the exoplanet's magnetosphere, to an exoplanet-induced signal into the star's magnetic field, or to exoplanet-independent stellar activity should be relatively easy on the basis of polarization and temporal modulations of the signal (see section 2.3). Thus measurements in circular (V-Stokes) or full polarization (4-Stokes) are required, over long or repeated observations for each target. Such measurements should be wealy affected by confusion, provided that the beam shape remains smooth and stable with time.

Experience from LOFAR, that uses elementary dipole antennas seeing a large fraction of the sky at any time, taught us that imaging will be more powerful than beamformed observations at least at the initial - detection - stage, because it allows to better remove the contribution of sources around the target and to obtain high dynamic range images, reaching a sensitivity of order of the thermal noise. Beamformed observations will be very useful to monitor - at much lower computational cost - the targets detected by imagery. New imaging techniques are also being developed, including predictor/estimator schemes such as Kalman filters (Tasse 2014), or decomposition of the imaging process on the Fourier transform of the observation time, that may considerably improve the efficiency of variable source imaging in the future.

\subsection{SKA Phase 1}

Jupiter bursts in the $30-40 \mathrm{MHz}$ range can exceed $10^{7} \mathrm{Jy}$ at Earth, i.e. $40 \mu \mathrm{Jy}$ at $10 \mathrm{pc}$ range, the average flux density of the emission at hour timescale being about one order of magnitude lower. SKA1-LOW, consisting of some 250000 dipoles in a radio-quiet site (Dewdney et al. 2013), will bring an improvement in sensitivity by a factor $\sim 10$ to 30 with respect to LOFAR in the range 50-250 MHz. SKA1-LOW will have a sensitivity $(1 \sigma)$ between 70 and $7 \mu \mathrm{Jy}$ across the band 50-350 MHz, with $4 \mathrm{MHz} \times 1$ hour integration (Figure 7). Confusion noise affecting deep images will be a few times larger in total intensity at the lowest frequencies (dashed line in Fig. 7, computed from (Condon 2005)). Detection of time-variable and/or circularly polarized emission will be less severely affected. Thus, Jupiter-like exoplanets will become detectable for the first time from distances of several parsecs. Even if bursts are intrinsically shorter, statistical accumulation (Ryabov et al. 2004) will permit to reach long integration times without diluting the signal.

Being little dependent on extrapolations of the scaling laws of section 2.2 to high-power ranges for reaching detectable emission levels (we will need emissions $\geq 10^{0-1} \times$ rather than $\geq 10^{3} \times$ Jupiter), and needing exoplanetary magnetic fields only slightly larger than Jupiter's to emit above $50 \mathrm{MHz}$, it is highly likely that SKA1-LOW will detect exoplanetary radio signals. 


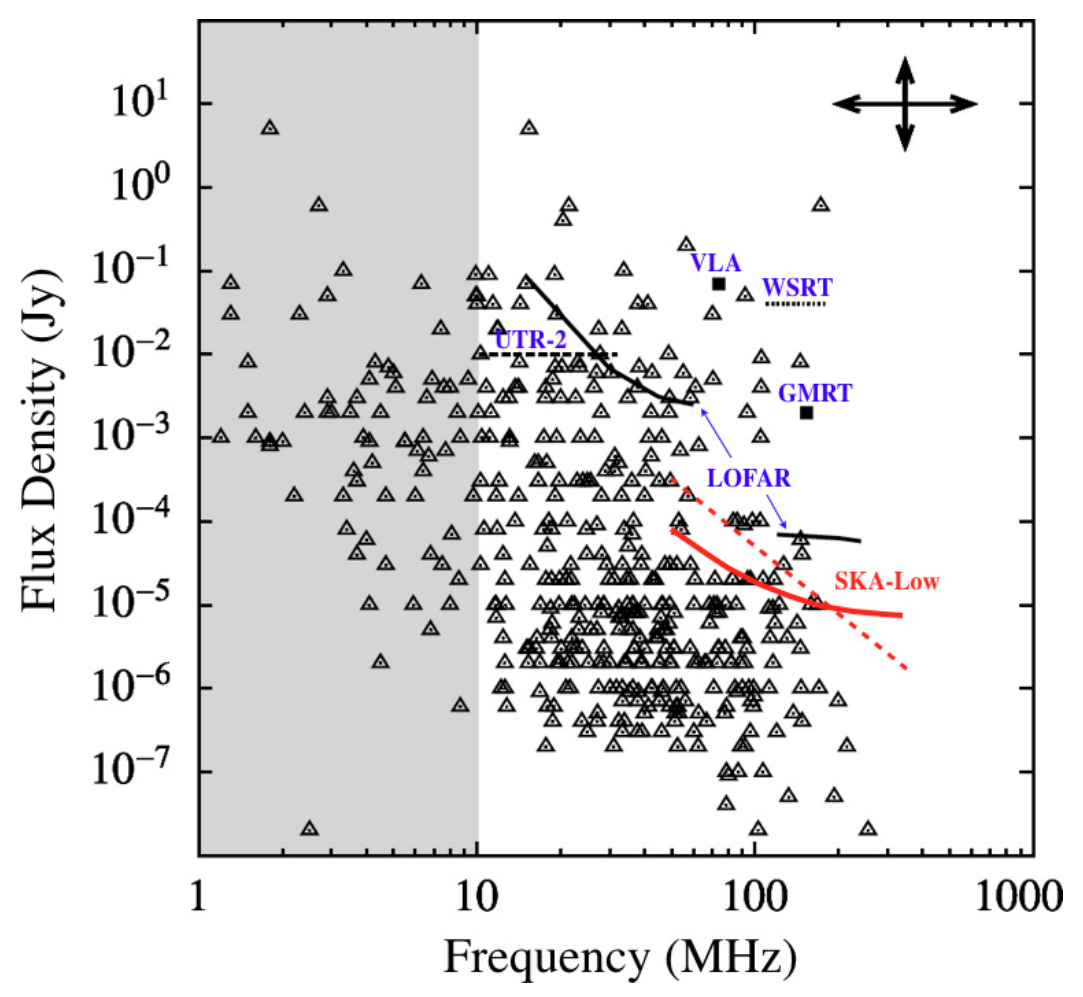

Figure 7: Predicted maximum emission frequency and expected radio flux for known exoplanets (in 2011, indicated by triangle symbols) for a rotation-independent planetary magnetic field and the scaling law of Fig. 2. The approximate sensitivity of several instruments is shown (for $1 \mathrm{~h}$ of integration time and a bandwidth of $4 \mathrm{MHz}$, or an equivalent combination). Confusion limit for SKA1-LOW is indicated by the dashed red line. Frequencies below $10 \mathrm{MHz}$ are not observable from the ground (ionospheric cutoff). Adapted from (Grießmeier et al. 2011).

But it remains correct to assume that exoplanets closer to their parent star, or orbiting stars with large X-UV flux, should emit stronger signals (e.g. $20 \times$ larger than Jupiter's for a Jovian-like exoplanet at $1 \mathrm{AU}$ from a solar-type star), and that unipolar inductor SPI do exist for exoplanets orbiting strongly magnetized stars. Thus a systematic search should reveal a large number of radioemitting exoplanets. These detections will give access to numerous measurements of exoplanetary magnetic fields, rotation periods, orbits inclinations, and SPI regimes and powers. They will open the field of comparative (exo-)planetary magnetospheric physics, may allow to discover moons around exoplanets (generating Io-Jupiter like CMI radiation), and will help to select favourable systems to study further in search for life signatures.

The search strategy should significantly evolve as compared to previous targeted searches. There are $\sim 400$ stars and white and brown dwarfs within $10 \mathrm{pc}$, out of which $\sim 200$ are known or observable, and $\sim 35$ exoplanets are currently known, and about 2500 known stars within $30 \mathrm{pc}$, with about 200 currently known planets. It will be possible with SKA to conduct an authoritative survey of all stars (visible from the SKA site) within $10 \mathrm{pc}$ and a large fraction of those within 30 pc, allowing to detect any radio emission $\sim 1$ to 10 times stronger than Jupiter's up to a distance of $10 \mathrm{pc}$, and any emission one order of magnitude stronger up to a distance of $30 \mathrm{pc}$. In parallel, all known exoplanets should be monitored ( $>1800$ planets are known today within $>1100$ systems 
- exoplanet.eu). Further emphasis may be put on systems for which the stellar magnetic field is measured and large, the phase of strongest radio signals being predictable in that case from stellar magnetic field extrapolations (Farès et al. 2010) (and unique information can thus be obtained from the comparison between observational results and predictions). Exoplanet-rich fields of view (e.g. deduced from Kepler observations and the new generation ESO-VLT instruments - see section 5.2) are also targets of particular interest, where it will be possible to search in long observations series any periodic signal matching an exoplanet's rotation or orbital period.

For each target, several observations of several hours each should be performed to account for phase effects (combined to the narrow beaming of CMI emission) or intrinsic variability. Maximum sensitivity is needed at all times, implying the use of the full array, and circular or full-polarization observations. Modest temporal and spectral resolutions are needed for meeting the physical objectives described above, typically $\delta \mathrm{f} / \mathrm{f} \simeq 1 \%$ (or $\sim 1 \mathrm{MHz}$ spectral channels) and $\delta \mathrm{t} \simeq 1 \mathrm{sec}$ to $1 \mathrm{~min}$. Finer resolutions are temporary required for the purpose of RFI mitigation before post-integration. The large number of targets to observe several times requires large duty cycles and large fields of view. In order to increase the SKA1-LOW FoV $\left(\sim 5 \times 5^{\circ 2}\right.$ at $110 \mathrm{MHz}$, scaling as $\left.\lambda^{2}\right)$, multibeam observations are very desirable. As emissions are more likely to occur at low frequencies, restricting the beam bandwidth (e.g. to $50 \mathrm{MHz}$ ) can allow to form many beams at little cost on the system capabilities. The availability of as many beams as possible will be a significant advantage for exoplanet search, as for searches or any other transient signals (exoplanetary radio emissions are slow transients).

Finally, all survey-type programs that will be conducted with SKA1-LOW during SKA Phase 1 (e.g. transients searches) should have their data correlated with the exoplanet catalog (exoplanet.eu), searching for coincidences (as for the GMRT and LOFAR sky surveys), that may guide follow-on observations. More generally, the search for emission at the coordinates of all known exoplanets should be systematically performed commensally to all SKA1-LOW observations.

\subsection{Early science operations of SKA Phase 1}

At a fraction $\sim 50 \%$ of SKA1-LOW capabilities, SKA1-LOW will already be a powerful instrument to detect radio emissions from exoplanets. There is thus interest to start intensive observations at an early phase of the project, concentrating on the most favourable targets among those listed above, i.e. stars within 5 to $10 \mathrm{pc}$, known exoplanets for which a large radio flux is predicted (Lazio et al. 2004, Grießmeier et al. 2007, 2011, Nichols 2012), or for which a hint of detection exist (Lecavelier et al. 2013, Sirothia et al. 2014), and known exoplanets orbiting strongly magnetized stars. The commensal searches mentioned in the previous section should be carried on from the beginning of the operations. More generally, advantage should be taken from synergies with observations at other wavelengths, as described in section 5.2 below, from the early phases of the project.

\subsection{Science outcome anticipated from SKA Phase 2}

The imaging observations in search for exoplanetary signals conducted in Phase 1 will be limited by confusion rather than by thermal noise. Confusion noise will actually be several tens to hundreds of $\mu \mathrm{Jy}$ across the SKA1-LOW band ((Condon 2005) and Fig. 7). Extending SKALOW to a less compact array configuration, with baselines e.g. up to $200 \mathrm{~km}$ as for SKA1-MID, 
would reduce the confusion to values close to the thermal noise (and at the same time reduce the thermal noise because of increased collecting area), down to a few $\mu \mathrm{Jy}$ in the range 50-350 MHz, making Jupiter-like emission easily detectable at $>10 \mathrm{pc}$ range. Increased field of view will improve systematic searches. The higher signal-to-noise ratio with which dynamic spectra of exoplanet's radio emissions will be measured, beyonf the few $\mathrm{dB}$ required for mere detection, will enable detailed quantitative interpretation of the observations in the frame of the simulations of (Hess \& Zarka 2011) (see section 2.3).

SKA2 will also extend and deepen large surveys started with SKA1. This will make increasingly relevant the systematic search of exoplanetary signals in these surveys by correlation with the exoplanet catalog. Increased angular resolution of the observations (to $\leq 3$ " at $110 \mathrm{MHz}$ ) will make coincidences more robust, less likely to be due to background radio sources. With the development of SKA1-SUR and SKA1-MID, searches for periodic higher frequency unipolar inductor emission such as predicted by Willes \& Wu $(2004,2005)$ should be targeted at known (magnetic) white dwarfs, following a strategy that will depend of the statistics of known white dwarf properties (known magnetic field, angular distribution, distance...). Multi-beam capability will undoubtly be very useful. More synergies will become relevant, with other SKA observations (section 5.1 below), as well as with observations at other wavelengths (section 5.2).

\section{Synergies}

\subsection{Synergies with other SKA observations}

Detection of stellar radio flares with SKA will have similar instrumental requirements as exoplanet radio detection (sensitivity, circular polarization, detection of bursts, large duty cycles). For example, SKA1-LOW will be able to detect the brightest solar bursts out to $\geq 10 \mathrm{pc}$.

But there are also deep physical connections between stellar physics and activity and exoplanet search:

- Stellar flares and mass ejections - such as solar CMEs and associated solar "type II" radio bursts - can excite magnetospheric planetary emissions (and cause atmospheric loss).

- Some stellar flares could actually be induced by an orbiting close-in exoplanet (through unipolar induction or magnetic reconnection).

- There is probably a continuum of physical conditions from brown dwarfs to exoplanets (decreasing rotational period, increasingly cool and neutral atmosphere, transition to large-scale stable magnetic field topologies, with weaker magnetic field magnitude). Circularly polarized periodic bursts have been discovered from the former at $\mathrm{GHz}$ frequencies (Hallinan et al. 2007, 2008), with brightness temperatures $>10^{15} \mathrm{~K}$. They have been attributed to CMI emission in kGauss magnetic fields. The duration of the bursts is a few percent of the recurrence period. This justifies the large duty-cycle required for their detection, and similarly for the detection of exoplanetary radio signals. Tracing magnetic fields and radio flux densities from brown dwarfs to planets will bring unique constraints for dynamo theories as well as for radio emission scaling laws. 
- Finally, Kepler has shown that lower mass planets are more frequent around M dwarfs (Dressing \& Charbonneau 2013), and close-in planets may be located in the habitable zone (although habitability may need to be redefined based on magnetic activity).

Provided that complex time domain voltage data from tied array beams can be exported in parallel with imaging or beamformed spectral observations, sensitive SETI searches could be commensally performed with exoplanetary observations, in spectral ranges little or not explored by past SETI searches (see corresponding chapter).

\subsection{Synergies with observations at other wavelengths}

As already mentioned above, measurements of stellar magnetic fields by Zeeman-Doppler imaging (with instruments such as CFHT/Espadons and TBL/Narval in visible light, and with CFHT/Spirou in the infrared in the near-future) are a key ingredient to assess SPI whose associated emission will be measured with SKA. Magnetic topology can influence the direction of emission of CMEs and in turn their impact on orbiting exoplanets. Measurements of large magnetic fields will also motivate SKA observations in search for unipolar inductor emissions, whose level can be in principle predicted along the planet's orbit (Farès et al. 2010). UV and X observations by HST, XMM-Newton, Chandra, and in the future by the Athena+ X-ray observatory will characterize stellar outputs and allow to identify best exoplanet candidates for enhanced magnetosphere-ionosphere coupling (Nichols 2011, 2012). Observations of large flares can also motivate specific searches of radio emissions triggered by these flares (in parallel with spectroscopic studies of their impact on exoplanet's atmosphere), as was done successfully to detect Uranus' aurorae (Lamy et al. 2012).

The number of known planets in the solar neighbourhood should increase substantially thanks to the missions PLATO (Planetary Transits and Oscillations of stars) and TESS (Transiting Exoplanet Survey Satellite), whereas Spirou will find Earth-like planets around nearby red dwarfs. The JWST will be able to characterize the atmospheres of these nearby planets, whereas magnetospheric studies with SKA will bring constraints to their habitability. Studies of lower mass planets will also provide insights to different regimes of planetary dynamos. New surveys by the ESOVLT instruments NGTS (Next-Generation Transit Survey) and ESPRESSO (Echelle SPectrograph for Rocky Exoplanet and Stable Spectroscopic Observations) will likely increase the number of confirmed exoplanets up to several tens per field of view of SKA1-LOW.

Finally, LOFAR will continue to provide observations within part of the SKA1-LOW range in the northern hemisphere, whereas the lower frequency ( $\leq 50 \mathrm{MHz}$ and down to $10 \mathrm{MHz}$ ) radiotelescopes UTR-2, NenuFAR, OLWA, will perform follow-on observations of SKA1-LOW candidates (in the overlapping part of the sky) or vice-versa.

\section{Conclusion}

Thanks to our experience of solar system magnetospheres and radio emissions, and extensive theoretical studies, the interpretation frame of exoplanetary radio observations is ready, waiting for the first confirmed detections and measurements of dynamic spectra. Beyond the measurement of several exoplanet's parameters, including the magnetic field (with implications on dynamo theory and habitability) and rotation, radio detections will open the new field of comparative exoplanetary 
magnetospheric physics and permit to study flow-obstacle plasma interactions in various speed, density, magnetic field, mass regimes, from hot jupiters to low-mass planets around (M) dwarfs.

Up to now, detecting the signal is the most difficult step. But the characteristics of SKA1-LOW (minimum frequency of $50 \mathrm{MHz}$, sensitivity of a few to a few $10 \mathrm{~s} \mu \mathrm{Jy}$, full Stokes measurements) will permit the detection of Jupiter like emission from $\geq 10 \mathrm{pc}$, independent of any extrapolation of solar system-based scaling laws (provided that Jupiter's magnetic field and radio flux density are not exceptionally high). Actually, scaling laws and theory suggest the existence of much stronger emissions, that will allow us to search much larger volumes of space.

The context of these searches will be favoured by the expected rapid growth in the number of known exoplanets due to new instruments and space missions (PLATO, TESS, JWST, NGTS, EXPRESSO...) that could result in tens of exoplanets per SKA1-LOW field of view. In order to take full advantage of this context, large duty-cycles are needed (repeated, hours-long observations), for which a multi-beam capability will be extremely useful. Observations will include targeted searches, commensal observations, and correlation of surveys to up-to-date exoplanet catalog. Radio flux densities of 1-10× Jupiter's will be detectable within $10 \mathrm{pc}$, where all stars should be observed. A large fraction of all stars within $30 \mathrm{pc}$ should also be observed, with a detection threshold one order of magnitude larger. All exoplanets should eventually be the subject of targeted searches, starting with systems with large stellar magnetic field or large X-UV flux.

Such synergies with observations at different wavelengths will be important, but also synergies with other SKA observations, especially concerning the "star-planet connection": a study of the field topology, dynamo, and radio output from dwarfs to planets. Eventually, radio detection may evolve as a discovery tool, e.g. adapted to finding planets (giant or terrestrial) around active, magnetic or variable stars. The realm that remains to explore is huge.

\section{Acknowlegments}

Part of this research was carried out at the Jet Propulsion Laboratory, California Institute of Technology, under a contract with the National Aeronautics and Space Administration. PZ thanks L. Lamy for useful comments.

\section{REFERENCES}

Berge, G. L. \& Gulkis, S. 1976, in Jupiter, T. Gehrels ed., Univ. Arizona Press, 621

Bigg, E. K. 1964, Nature, 203, 1008

Burke, B. F., \& Franklin, K. L. 1955, J. Geophys. Res., 60, 213

Condon, J. J. 2005, ASPC 345: From Clark Lake to the LWA, N. E. Kassim et al. eds., 237

de Pater, I. 2004, Planet. Space Sci., 52, 1449

Dewdney, P., Turner, W., Millenaar, R., et al. 2013, SKA1 System Baseline Design, Document number SKA-TEL-SKO-DD-001, Rev. 1

Dressing, C. D., \& Charbonneau, D. 2013, Astrophys. J., 767, 95

Farès, R., Donati, J.-F., Moutou, C., et al. 2010, MNRAS, 406, 409

Farrell, W. M., Desch, M. D., \& Zarka, P. 1999, J. Geophys. Res., 104, 14025 
Farrell, W. M., Desch, M. D., Lazio, T. J., et al. 2003, ASPC 294: Scientific Frontiers in Research on Extrasolar Planets, 151

Farrell, W. M., Lazio, T. J., Desch, M. D., et al. 2004, IAUS 213, Bioastronomy 2002: Life Among the Stars, R. Norris \& F. Stootman eds., ASP, 73

George, S. J., \& Stevens I. R. 2007, MNRAS, 382, 455

George, S. J., \& Stevens, I. R. 2008, MNRAS, 390, 741

Grießmeier, J.-M., Stadelmann, A., Penz, T., et al. 2004, Astron. Astrophys., 425, 753

Grießmeier, J.-M., Stadelmann, A., Motschmann, U., et al. 2005, Astrobiology, 5(5), 587

Grießmeier, J.-M., Zarka, P., \& Spreeuw, H. 2007, Astron. Astrophys., 475, 359

Grießmeier, J.-M., Zarka, P., \& Girard, J. N. 2011, Radio Science, 46, RS0F09

Jardine, M., \& Cameron, A. C. 2008, Astron. Astrophys., 490, 843

Hallinan, G., Bourke, S., Lane, C., et al. 2007, Astrophys. J., 663, L25

Hallinan, G., Antonova, A., Doyle, J. G., et al. 2008, Astrophys. J., 684, 644

Hallinan, G., Sirothia, S. K., Antonova, A., et al. 2013, Astrophys. J., 762, 34

Hartman, J., Hallinan, G., Taylor, G., et al. 2013, LWA Users Meeting, Arlington, VA

Heald, G., \& the MSSS team 2014, LOFAR science meeting, Amsterdam, www.astron.nl/lofarscience2014/

Hess, S. L. G., \& Zarka, P. 2011, Astron. Astrophys., 531, A29

Lamy, L., Prangé, R., Hansen, K. C., et al. 2012, Geophys. Res. Lett., 39, L07105

Lazio, T. J. W., Farrell, W. M., Dietrick, J., et al. 2004, Astrophys. J., 612, 511

Lazio, T. J. W., \& Farrell, W. M. 2007, Astrophys. J., 668, 1182

Lazio, T. J. W., Shankland, P. D., Farrell, W. M., et al. 2010a, Astron. J., 140, 1929

Lazio, T. J. W., Carmichael, S., Clark, J., et al. 2010b, Astron. J., 139, 96

Lecavelier des Etangs, A., Sirothia, S. K., Gopal-Krishna, et al. 2009, Astron. Astrophys., 500, L51

Lecavelier des Etangs, A., Sirothia, S. K., Gopal-Krishna, et al. 2011, Astron. Astrophys., 533, A50

Lecavelier des Etangs, A., Sirothia, S. K., Gopal-Krishna, et al. 2013, Astron. Astrophys., 552, A65

Nichols, J. D. 2011, MNRAS, 414, 2125

Nichols, J. D. 2012, MNRAS, 427, L75

Ryabov, V. B., Zarka, P., \& Ryabov, B. P. 2004, Planet. Space Sci., 52, 1479

Shkolnik, E., Bohlender, D. A., Walker, G. A. H., et al. 2008, Astrophys. J., 676, 628

Sirothia, S. K., Lecavelier des Etangs, A., Gopal-Krishna, et al. 2014, Astron. Astrophys., 562, A108

Tasse, C. 2014, Astron. Astrophys., 566, A127

Treumann, R. 2006, Astron. Astrophys. Rev, 13, 229

van Haarlem, M. P., \& the LOFAR collaboration 2013, Astron. Astrophys., 556, A2

Willes, A. J., \& Wu, K. 2004, MNRAS, 348, 285

Willes, A. J., \& Wu, K. 2005, Astron. Astrophys., 432, 1091

Winterhalter, D., Kuiper, T., Majid, W., et al. 2006, in Planetary Radio Emissions VI, H. O. Rucker et al. eds., Austrian Acad. Sci. Press, 595

Zarka, P., Queinnec, J., Ryabov, B. P., et al. 1997, in Planetary Radio Emissions IV, H. O. Rucker et al. eds., Austrian Acad. Sci. Press, 51 
Zarka, P. 1998, J. Geophys. Res., 103, 20159

Zarka, P., Treumann, R. A., Ryabov, B. P., et al. 2001, Astrophys. Space Sci., 277, 293

Zarka, P. 2004, Planet. Space Sci., 52, 1455

Zarka, P. 2007, Planet. Space Sci., 55, 598

Zarka, P. 2010, in Pathways Towards Habitable Planets, ASPC 430, V. Coudé du Foresto et al. eds., 175

Zarka, P. 2011, in Planetary Radio Emissions VII, H. O. Rucker et al. eds., Austrian Acad. Sci. Press, 287 